Chirurg 2019 · 90 (Suppl 2):S121 https://doi.org/10.1007/s00104-019-0828-1 Online publiziert: 13. Februar 2019 (c) Springer Medizin Verlag $\mathrm{GmbH}$, ein Teil von Springer Nature 2019

\section{Originalpublikation}

Park AE, Zahiri HR, Hallbeck MS et al (2017) Intraoperative "micro breaks" with targeted stretching enhance surgeon physical function and mental focus: a multicenter cohort study. Ann Surg 265:340-346

Hintergrund. Chirurgen sind im Operationssaal einer hohen geistigen und körperlichen Belastung ausgesetzt. Berufsbedingte Schmerzen, die häufig aus dem Zusammenspiel von unphysiologischer Körperhaltung und statischer Muskelbelastung resultieren, sind eine der häufigsten Ursachen für Krankheitsausfall und vorzeitigen Ruhestand. In der vorliegenden Studie wurde der Einfluss kurzer intraoperativer Dehnungspausen (TSMB, „targeted stretching micro breaks") auf Schmerzen sowie physische und psychische Leistungsfähigkeit untersucht.

Methoden. An vier namhaften US-amerikanischen Zentren beurteilten Chirurgen und Operationspersonal an zwei Operationstagen Schmerzen, Müdigkeit sowie geistige und körperliche Leistungsfähigkeit. An einem der beiden Operationstage wurden keine TSMB durchgeführt, am anderen Operationstag erfolgten zu geeigneten Zeitpunkten in Abständen von 20 bis 40 min standardisierte 1,5- bis 2-minütige angeleitete TSMB.

Ergebnisse. Insgesamt 66 Teilnehmer (69\% Männer, $31 \%$ Frauen, Durch-

Dieser Beitrag wurde erstpubliziert in Der Chirurg (2017) 88:256-256. https://doi.org/10. 1007/s00104-017-0391-6

\title{
Intraoperative Mikropausen reduzieren Schmerzen und verbessern die Konzentration
}

schnittsalter 47 Jahre) führten 193 Operationen ohne TSMB und 148 Operationen mit TSMB durch. $47 \%$ der Chirurgen hatten Sorge, dass Schmerzen ihre Karriere verkürzen könnten, und $67 \%$ erachteten es als Notwendigkeit, trotz Schmerzen weiter zu arbeiten, um ihr Operationstagesprogramm zu erledigen. Komplexität und Schwierigkeitsgrad der Eingriffe sowie die durchschnittliche Operationsdauer unterschieden sich nicht zwischen den Gruppen mit und ohne Mikropausen. TSMB führten zu einer signifikanten Reduktion von Schmerzen im Bereich des Nackens $(p=0,01)$, der Schultern $(p<0,001)$, der Hände $(p=$ $0,03)$ und des LWS-Bereichs $(p=0,03)$. $57 \%$ der Teilnehmer empfanden eine gesteigerte körperliche Leistungsfähigkeit und $38 \%$ berichteten über eine bessere Konzentration durch TSMB. 87\% der Chirurgen wollten TSMB in Zukunft weiter durchführen.

Diskussion und Fazit. Chirurgen sind teilweise extremen Belastungen ausgesetzt. Auch laparoskopische Eingriffe stellen manchmal aufgrund von Fulcrum-Effekt und ungünstigen Arbeitspositionen eine besondere Herausforderung dar. Engelmann et al. zeigten, dass Mikropausen die Rate an intraoperativen Ereignissen und Fehlern reduzierten, ohne die Operationsdauer zu verlängern [1]. Damit haben Pausen möglicherweise einen direkten Einfluss auf die Qualität der chirurgischen Versorgung. Die vorgestellte Studie zeigte auch, dass sich die Teilnehmer häufig nicht in Behandlung begaben und trotz Schmerzen ihre Arbeit verrichteten - eine auch in Deutschland stereotypische Haltung von Chirurgen. Chirurgen stellen im Allgemeinen eine hochmotivierte Berufsgruppe dar, die sich mit Hingabe der Patientenversorgung widmet und dazu neigt, eigene, berufsbedingte Befindlichkeitsstörungen $\mathrm{zu}$ bagatellisieren. Viele Unternehmen investieren heute große Summen in eine ergonomische Arbeitsplatzgestaltung und in Gesundheitsprogramme, um die Produktivität qualifizierter Mitarbeiter auf hohem Niveau zu halten. Diese Denkansätze sind in den unseren Krankenhäusern bislang nur selten zu finden. Zum einen mangelt es grundsätzlich an der Wahrnehmung dieses Problems bzw. es besteht kein Interesse, Leistungseinbußen sowie krankheitsbedingte Fehlzeiten hochqualifizierten Personals zu verringern. Die hier vorgestellte Arbeit zeigt, wie mit einfachen Mitteln die körperliche Belastung im Operationssaal und deren Folgen, die dieser einzigartige Beruf mit sich bringt, reduziert werden können.

\section{Korrespondenzadresse}

\section{Prof. Dr. M. Anthuber}

Klinik für Allgemein-, Viszeral- und

Transplantationschirurgie, Klinikum Augsburg Stenglinstr. 2, 86156 Augsburg, Deutschland matthias.anthuber@klinikum-augsburg.de

Interessenkonflikt. M. Schrempf und M. Anthuber geben an, dass kein Interessenkonflikt besteht.

\section{Literatur}

1. Engelmann C, Schneider M, Kirschbaum $C$ et al (2011) Effects of intraoperative breaks on mental and somatic operator fatigue: A randomized clinical trial. Surg Endosc 25:1245-1250 